Lang, A., Hignett, S. (2018) Ergonomics/Human Factors in Healthcare: a vision for the future. Proceedings of the $20^{\text {th }}$ Triennial Conference of the International Ergonomics Association. Florence, Italy, 2630 August.

\title{
Ergonomics/Human Factors in Healthcare: a vision for the future
}

\author{
Alexandra Lang ${ }^{[0000-0002-7332-9443]}$ and Sue Hignett ${ }^{[0000-0002-3025-7451]}$ \\ ${ }^{1}$ MindTech, University of Nottingham, UK; Chair of Special Interest Group in Healthcare \\ Ergonomics, Chartered Institute of Ergonomics \& Human Factors \\ ${ }^{2}$ Loughborough Design School, Loughborough University, UK; Chair of Professional Affairs \\ Board, Chartered Institute of Ergonomics \& Human Factors \\ Alexandra/Lang@nottingham.ac.uk; S.M.Hignett@lboro.ac.uk
}

\begin{abstract}
How could and should Human Factors/Ergonomics (HFE) be implemented in healthcare? The Chartered Institute of Ergonomics \& Human Factors (CIEHF, UK) has developed a vision for the future to complement the strategic views expressed by the Care Quality Commission, NHS Education for Scotland (NES), Health Education England (HEE), Medicines and Healthcare products Regulatory Agency (MHRA) and others. The initiatives delivered in the National Health Service (NHS, UK) have succeeded in sparking an interest in HFE. Whilst there have been successes in introducing local improvements using risk management, quality improvement and patient safety methods, the participative nature of HFE supports collaboration by all stakeholders ensuring that a system-wide approach is taken. This offers a new beginning for a common understanding of HFE in healthcare, accessible to all healthcare service stakeholders and provides a vision for education, capacity building and implementation. This paper will describe the consultation process and give examples of 'what good looks like'; for example making the best use of human capabilities (physical, cognitive, psychological and social characteristics); mitigating for human limitations; and utilizing people in ways that maximize system safety and minimize risk.
\end{abstract}

Keywords: Healthcare, Vision, Integration

\section{Introduction}

Human Factors/Ergonomics (HFE) input in any industrial sector often follows a major incident or change in legislation. In the 1990s, the removal of Crown Immunity from prosecution under the Health and Safety Act 1974 meant that the NHS had to comply with safety legislation as hospitals and other care locations were considered to be places of work. From the 1980s to 2000s, HFE input focused on occupational health [1], building design [2], and systems approaches to embed HFE as part of the health care organizational culture [3].

In 2004 the National Patient Safety Agency (NPSA) recommended the use of HFE as part of the 'Seven Steps to Patient Safety' [4]. There has been much good progress 
in the last 14 years including a National Concordat [5] bringing together 16 organisations. Health and social care has already started to benefit from HFE approaches and there will be many opportunities in the future. However, although the need for HFE in healthcare has been recognised the development and growth have been slow. We suggest that, in the UK, this is partly due to a misconception about what HFE is. The driving influences for 'Human Factors' in the UK have been from academic discipline of organisational (or industrial) psychology [6] and the industrial aviation sector [7]. There was a lack of engagement with the UK HFE professional body (Chartered Institute of Ergonomics \& Human Factors, CIEHF) which, in our opinion, contributed to a dysfunctional separation (or lack of integrated safety) for the human elements into occupational health for staff, and patient safety [8]. This response is similar to that for occupational musculoskeletal problems where, for many years guidance from clinical professional bodies focused on technique (behavior) training. However, after 20 years, there was strong research evidence that 'interventions predominantly based on technique training had no impact on working practices or injury rates'[9] and the focus moved to a Systems approach [3].

Catchpole [10] commented that 'while entirely legitimate and increasingly well evidenced, [this behavioural safety approach] is limited.... Frequently espoused by wellmeaning clinicians and aviators, rather than academically qualified HF professionals, it has led to misunderstandings about the range of approaches, knowledge, science and techniques that can be applied from the field of HF to address patient safety and quality of care problems'. Russ et al [11,12] suggested that these influences have contributed to the tendency to blame 'the failures of people as the underlying cause of adverse events or broken healthcare delivery processes, a stance that is contrary to human factors science and counterproductive for advancing patient safety' as 'little attempt is made to explore and address the underlying systemic causes that lead to errors.'

This paper describes the process to develop a professional White Paper as a vision for future HFE integration in health and social care in the UK. The White Paper is aimed at health and social care service managers and Human Factors champions in care settings, as well as anyone interested in ensuring health and social care is the best it can be.

\section{Developing the White Paper}

The need for a White Paper to address the misconceptions has previously been discussed with respect to quality improvement [13]. In 2016, a workshop was held to explore how HFE knowledge and experience in defence could both inform and possibly be transferred to healthcare [14]. It was felt that there was a better 'fit' between defence and healthcare rather than the usual comparison between aviation and healthcare. Both defence and healthcare have complex and potentially chaotic working environments; activities take place 24 hours a day, 365 days a year; and there are professional 'silos' (e.g. army, navy, air force). The workshop resulted in a 1, 5, 10 and 20 year principles (framework for a route map) including: 
1. Service level professional collaboration agreements with partner organisations

2. Clear message and aim with plan for way forward

3. Examples as case studies (and/or safety cases)

4. Competency matrix

5. HFE applied in investigations (local incident reports through to Coroner's Court)

6. HFE and Quality Improvement linked

7. HFE included in Care Quality Commission inspections

8. HFE mandatory in processes for audit, procurement etc.

9. HFE as uniform approach across all Trusts, sectors (primary, secondary, mental health, ambulance, community \& home care)

10. HFE competency matrix - clear, embedded and audited

11. HFE capacity to deliver improved safety

It was proposed that, by 2036 HFE should be mandatory in healthcare processes for audit, procurement and so forth with a uniform approach across all healthcare providers as in defence [15]. The role of HFE providers (SQEP; Suitably qualified/experienced person) will be clearly defined based on CIEHF professional competencies to provide assurance in the same way as other professional regulators (e.g. General Medical Council, Nursing and Midwifery Council, General Pharmaceutical Council).

\subsection{White Paper project proposal}

A Project Initiation Document was submitted to the Chartered Institute of Ergonomics \& Human Factors (CIEHF) Council in 2017 with a proposal to develop the CIEHF vision for Human Factors/Ergonomics (HFE) in Healthcare as the UK authoritative guide to help readers understand how HFE could and should be used. This included:

- HFE best practice in processes including audit, investigation and procurement from e.g. local pharmacy services and medical devices to national inter-agency information communication systems across all service providers (public and private), and sectors (primary, secondary, mental health, ambulance, community, social and home care).

- Strategies for HFE Implementation (e.g. integration with quality improvement) including a sector specific competency matrix

- HFE capacity to deliver improved safety as a resilient system for safety culture and work load

- Education including undergraduate curricula for Pharmacy, Academy of Medical Royal Colleges and postgraduate training

- Competencies and experience needed to fill the various roles required for effective HFE implementation.

The project was announced at the CIEHF annual conference 2017 to explore interest and start the development/engagement process as part of a healthcare ergonomics symposium. 


\subsection{Workshop to develop key messages}

The purpose of the workshop was to agree key messages and bring together source material. A wide range of participants included CIEHF members representing most grades (Associate, Graduate, Registered, Fellow); Chairs of two CIEHF Special Interest Groups (Healthcare and Pharmaceutical), current and previous representatives of the Healthcare Safety Investigation Branch [16] and National Patient Safety Agency [17] and input from NHS Education Scotland [18], Clinical Human Factors Group (advocate) [19].

The possible scope of the White Paper was discussed in detail including:

- HFE best practice in processes and design

- All service providers (public and private), and sectors (primary, secondary, mental health, ambulance, community, social and home care)

- Boundaries, e.g. exclude pharmaceutical manufacturing pipeline

- What HFE is and is not.....?

- Strategies for Implementation including competency and experience the various HFE roles.

\section{The White Paper}

\subsection{Vision statement}

The draft vision statement is that 'through ongoing collaboration, co-creation and discovery with health and social care providers, professionally qualified Human Factors Specialists will contribute towards developing and embedding sustainable system-level improvements'. It is proposed that this will be achieved by:

- Building on existing good work

- Broadening the scope of Human Factors understanding

- Guiding the understanding of shared aims and offerings from partner organisations

- Promoting the integration of Human Factors to optimise human (patient and staff) wellbeing and overall system performance

- Raising awareness of the discipline as an accredited, professional career

- Ensuring and maintaining the standard of Human Factors practice through demonstration of competence and experience

- Encouraging the contribution of professional (qualified) Ergonomists \& Human Factors Specialists via consultation and employment

- Championing an accessible, user-focused approach.

\subsection{What is Human Factors}

This section provides the IEA definition [20] and dispels myths about differences between Human Factors and Ergonomics. 'Systems' are introduced as a fundamental HFE concept with examples for micro and macro systems. 
Ideas for integrating HFE into healthcare systems include ensuring HFE practice is design-led and proactive; working with existing risk management programmes (including quality improvement); and being creative in developing inclusive solutions so that products and services will be accessible to, and usable by, as many people as reasonably possible.

\subsection{Increasing Human Factors Competency and Capacity}

HFE awareness already exists for a range of professionals in healthcare including psychologists, occupational therapists, physiotherapists, medical device designers and engineers. So, part of the vision is to increase Human Factors competence with, for example, inclusion of HFE content in clinical curricula. The World Health Organization [21] developed a patient safety curriculum, but little is known about how providers ensure learners develop safety competencies. A set of 12 tips has been proposed for incorporating HFE principles and methods in clinical curricula to enhance the effectiveness of safety and improvement work in frontline healthcare practice [22]. The 12 tips include the systems framework, HFE tools and competency, and ideas for implementation.

Professional behaviour in HFE is guided by the CIEHF Code of Professional Conduct [23] and professional competencies [24] in 5 domains with 6 levels of competency (Fig. 1).

1. Unaware: No knowledge or understanding of this competency.

2. Aware: Knowledge or an understanding of basic techniques and concepts particular competency for a particular competency.

\$. Novice: Limited experience gained in a classroom and/or as a trainee on-the-job for a particular competency

4. Intermediate: Can successfully complete tasks independently in a particular competency; can demonstrate the appropriate use of different techniques and methods in the application of Human Factors research or consultation.

\$. Advanced: Can perform actions associated with this competency without assistance; can bring together disparate theories and techniques or the application of novel solutions to complex problems

\$. Expert: Recognised authority in this area; can provide guidance, troubleshoot and answer questions related to this area of expertise with consistent excellence in applying this competency across multiple projects and/or organisations.

Fig. 1. Proficiency levels for Professional Competencies

Ideas for increasing capacity include HFE content in clinical curricula, input from qualified HFE professionals; recommending that every health and social care organisation has an identified Human Factors advisor at a senior level. The goal is to develop sufficient HFE capability to deliver a resilient system that encompasses safety culture and acceptable workloads for all healthcare providers. 


\subsection{Understanding how to use Human Factors}

Three areas are covered for understanding how to use HFE. These are (1) using HFE in investigations; (2) thinking about systems; and (3) thinking about design. For investigations, where human error may seem at first to be the cause of an incident, an HFE approach takes a wider view to encompass contributing factors such as poor product design. For example an interim report by HSIB [25] has identified systems issues including different processes for portable and installed systems (1-2 step tasks) and lack of visual indicators (feedback) for both flow and residual volume.

The Systems of Systems in healthcare have been described as 'nested and overlapping systems; ' $a$ bed in a hospital is a system, the patient monitoring equipment is a sibling system, the two together plus the patient's room comprise another system, ...; whereas the radiology or scanning equipment, the drugs dispensary, the beds, the ambulances are all systems, but together can be seen as a system of systems when looking at maintenance and replacement regimes' [26].

HFE is relevant at all stages (upstream and downstream) in the life-cycle of equipment, environment and services; from early stages of planning and design, right through to implementation and evaluation, and re-design. Healthcare is starting to recognise the benefits of HFE where the systems view of safety and an inclusive, human-centred design process can be applied in nearly all work situations.

\subsection{Implementing Human Factors}

Health and Social Care organisations face challenges in embedding and sustaining improvement initiatives. Successful implementation often relies on individuals having sufficient knowledge, time and resources to effectively play their part.

The vision for Human Factors implementation is:

- Human Factors good practice is common across all health and social care processes including audit, new and redesigned services, investigation and procurement.

- Human Factors good practice informs all areas from local pharmacy services and medical devices to national inter-agency information communication systems across all service providers (public and private), and sectors (primary, secondary, mental health, ambulance, community, intermediate, social and home care).

- Proven strategies and frameworks exist and are used for Human Factors implementation, such as integration with Quality Improvement, including a sector-specific competency matrix to which all practitioners adhere.

- International standards for Ergonomics and Human Factors are embedded in healthcare design and systems for planning, acquisition and safety.

- The underlying culture is a learning culture, keen to drive continuous improvement in human performance and wellbeing. 


\subsection{Case Study example: Design for Patients}

Birth in water gained momentum in the early 1990s however the design of birthing pools at that time had not been designed with users in mind. Mothers found it difficult to get into the birthing pool and almost impossible to get out of in an emergency. Midwives had to adopt poor posture when monitoring and examining mothers in the pool. User needs were identified and incorporated into a new design which included steps and rails to assist entry and exit, shaped edges to support the mother, knee room to allow the midwife easier access for monitoring and a seat that allowed the mother to be evacuated rapidly in an emergency. The work revolutionised the design of birthing pools with resultant improvements in safety and wellbeing of the mother, baby and midwife. Support staff carrying out maintenance, cleaning and infection control also benefited from the improved design [27].

\section{Conclusion}

The White Paper is going through the final consultation stages (May 2018). Through ongoing collaboration, co-creation and discovery involving clinicians, HFE experts and other professionals within and beyond healthcare, this approach will contribute towards developing, and implementing sustainable system level improvements.

\section{References}

1. Straker, L. M. Work-Associated Back Problems: Collaborative Solutions. Occupational Medicine 40: 75-79. (1990).

2. Hilliar, P. The DHSS Ergonomics Data Bank and the Design of Spaces in Hospitals. Applied Ergonomics 12 (4): 209-216. (1981).

3. Hignett, S. Embedding Ergonomics in Hospital Culture: Top-down and Bottom-up Strategies. Applied Ergonomics 32: 61-69. (2001).

4. http://www.nrls.npsa.nhs.uk/resources/collections/seven-steps-to-patientsafety/?entryid45=59787, last accessed 2018/4/12

5. https://www.england.nhs.uk/wp-content/uploads/2013/11/nqb-hum-fact-concord.pdf, last accessed 2018/4/12

6. Flin, R., Maran, N. Identifying and training non-technical skills in acute medicine. Quality and Safety in Health Care, 13 (Suppl I), i80-i84. (2004)

7. Flin, R., Martin, L., Goeters, K., Hoermann, J., Amalberti, R., Valot, C. \& Nijhuis, H. Development of the NOTECHS (Non-Technical Skills) system for assessing pilots' CRM skills. Human Factors and Aerospace Safety, 3, 95-117. (2003).

8. Hignett, S., Carayon, P., Buckle, P. and Catchpole, K. State of science: human factors and ergonomics in healthcare. Ergonomics 56:1491-1503. (2013).

9. Hignett, S. Intervention strategies to reduce musculoskeletal injuries associated with handling patients: A systematic review. Occupational and Environmental Medicine. 60, 9, e6. (2003)

10. Catchpole, K. Spreading Human Factors expertise in Healthcare: Untangling the knots in People and Systems. BMJ Quality \& Safety 22:793-797. (2013). 
11. Russ, A.L., Fairbanks, R.J., Karsh, B-T., Militello, L.G., Saleem, J.J. and Wears, R.L.. The Science of Human Factors: separating Fact from Fiction. BMJ Quality \& Safety. 22:802808. (2013)

12. Russ, A.L., L.G.Militello, J.J. Saleem, et al. Response to separating Fact from Opinion: a Response to 'the science of Human Factors: separating Fact from Fiction. BMJ Quality \& Safety 22:964-966. (2013).

13. Hignett, S, Jones, E., Miller, D., Wolf, L., Modi, C., Shahzad, MW, Banerjee, J., Buckle, P., Catchpole, K. Human Factors \& Ergonomics and Quality Improvement Science: Integrating Approaches for Safety in Healthcare. BMJ Quality and Safety 24:250-254 (2015.)

14. Hignett, S., Tutton, W., Tatlock, K. Human Factors Integration (HFI) in UK Healthcare Route map for 1 year, 5 years, 10 years and 20 years In Charles, R. \& Wilkinson, J. (Eds) CONTEMPORARY ERGONOMICS 2017; PROCEEDINGS OF THE ANNUAL CONFERENCE OF THE CHARTERED INSTITUTE OF ERGONOMICS \& HUMAN FACTORS. Taylor \& Francis, London. (2017)

15. MOD. 2015. JSP 912. Human Factors Integration for Defence Systems. Part 1: Directive. https://www.gov.uk/government/uploads/system/uploads/attachment_data/file/483176/201 50717-JSP_912_Part1_DRU_version_Final-U.pdf, last accessed 2018/4/12

16. https://www.hsib.org.uk/, last accessed 2018/4/10

17. http://www.npsa.nhs.uk/, last accessed 2018/4/10

18. https://learn.nes.nhs.scot/800/patient-safety-zone/human-factors, last accessed 2018/4/10

19. https://chfg.org/, last accessed 2018/4/10

20. IEA, 2001. International Ergonomics Association. Core competencies. www.iea.cc/project/PSE\%20Full\%20Version\%20of\%20Core\%20Competencies\%20in\%2 0Ergonomics\%20Units\%20Elements\%20and\%20Performance\%20Criteria\%20October\%2 02001.pdf, last accessed 2018/4/11

21. WHO. 2011. World Health Organisation The Multi-Professional Patient Safety Curriculum Guide. http://www.who.int/patientsafety/education/curriculum/en/. last accessed 2017/6/15

22. Vosper, H., Bowie, P., Hignett, S. (2017) Twelve tips for embedding Human Factors and Ergonomics principles in healthcare educational curricula and programmes Medical Teacher https://www.tandfonline.com/doi/abs/10.1080/0142159X.2017.1387240

23. CIEHF Charter Documents, Professional Code of Conduct (page 20) https://www.ergonomics.org.uk/Public/About_Us/CIEHF_Documents/Public/About_Us/C IEHF_Documents.aspx?hkey=8df03a4a-ab8a-482d-8a50-99c4a052f0c7 last accessed 2018/4/11

24. CIEHF Professional Competencies https://www.ergonomics.org.uk/Public/Membership/Registered_Member/Public/Members hip/Registered_Member.aspx?hkey=32fc9cb9-6d12-45fd-a3cb-8063e4c256f4 last accessed 2018/4/11

25. https://www.hsib.org.uk/investigations-cases/design-and-safe-use-portable-oxygensystems/interim-bulletin/ last accessed 2018/4/13

26. Wilson, J. R. Fundamentals of Systems Ergonomics/Human Factors. Applied Ergonomics 45: 5-13. (2014).

27. Case Study 18: Improving birthing pool design. The Human Connection. CIEHF 2015 https://www.ergonomics.org.uk/Public/Resources/Publications/Human_Connection/Public /Resources/Publications/Case_Studies.aspx?hkey=6cef60ed-99a9-498e-b2b7edd072ff31dc last accessed 2018/4/13 